\title{
Research and Application of Noise Blind Mixed Signal On-line Separation Algorithm Based on ICA
}

\author{
Liu Fangyu*
}

Taiyuan University of Technology, Shanxi 030024, China

\begin{abstract}
Independent Component Analysis (ICA) is a kind of novel multi-dimensional statistical analysis method. Its characteristic is to recover the independent source signal components from the multi-channel observation signal. In this paper, based on the model of independent component analysis, the gradient style algorithm was used to separate the noise blind mixed electrical signal. In the application of gradient algorithm, in order to solve the compromise between convergence rate and stability performance of the natural gradient blind source separation algorithm, the variable step size natural gradient algorithm was adopted to complete the noise electrical signal on-line blind source separation simulation, which provides an effective method to realize the multi-sensor collection signal.
\end{abstract}

Keywords: Adaptive variable step size, Blind source separation, ICA, Natural gradient.

\section{INTRODUCTION}

Blind source separation (BSS) [1] is a new technology with rapid development in the late 1980's, which is used to separate each source signal from blind mixed signals, and is presented for a powerful signal processing method combining the neural network with the information processing theory. Now, blind source separation technology has been adopted in many fields such as biomedical, communication, speech, image processing, radar and communication systems, seismic exploration, mobile communications, etc. It has become an international research focus in the field of signal processing and artificial neural network, which has very important practical value. BSS algorithm mainly includes Informax, natural gradient algorithm, Fast ICA algorithm, etc [2]. Blind separation algorithm is based on information theory and higher order statistics theory to establish the cost function, and is used to optimize the objective function. Among the algorithms, the Fast ICA algorithm has faster rate of convergence and has been applied in many engineering fields; however, as it is a kind of batch algorithm, it cannot achieve on-line blind source separation. The gradient algorithm is a classical unconstrained optimization algorithm with simple principle, which is easy to implement and can realize the on-line calculation.

Due to the step size parameter of traditional natural gradient, algorithm is a fixed value; the fixed step size parameter cannot solve the contradiction between the rate of convergence and the steady-state error. If the value of step size is improved, the rate of convergence can be improved theoretically; however, it may lead to the steady state error and may even cause the divergence in algorithm [3]. This article is based on gradient blind source separation method which

*Address correspondence to this author at the No. 79 of Yingze West Street, Taiyuan, China, Postcard: 030024; E-mail: liufangyu2003@163.com comprehensively considers the computational complexity of algorithm, the rate of convergence and steady state performance. A kind of adaptive variable step natural gradient algorithm has been applied to the noise signal blind source separation, and comparing with the traditional blind source separation algorithm, satisfactory separation results have been achieved.

\section{MATHEMATICAL MODEL OF BLIND SOURCE SEPARATION}

BSS is a technology of separating independent source signals from the mixed signal under the transfer function, mixed coefficient of source signals and probability distribution of an unknown system [4]. Usually, signals from several vibration sources are measured through a certain number of sensors; each sensor measures the mixed signal source, but does not know the confusion matrix style of the nonlinear system. In this case, the analysis and assessment of the signal of each source are carried out separately. In the traditional blind source separation algorithm, firstly, $m$ numbers of signal sources and sensors are chosen; there exists the following formula between the measure signals and source signals:

$x(t)=A s(t)+v(t)$

In formula (1), the observation signal $x(t)=$ $\left[x_{1}(t), x_{2}(t), \ldots, x_{m}(t)\right]^{T}$, which is the instantaneous linear mixed stack of $n$ numbers with unknown source signal $s(t)$, $s(t)=\left[s_{1}(t), s_{2}(t), \ldots, s_{n}(t)\right]^{T}, s_{i}(t)(i=1,2, \ldots, n)$ is $n$ numbers random signal independent of each other, aliasing matrix A is $m \times n$ unknown mixed matrix, $A=\left(a_{i j}\right), i=1,2, \ldots, m ; j=$ $1,2, \ldots, n ; v(t)=\left[n_{1}(t), n_{2}(t), \ldots, n_{m}(t)\right]^{T}$ is $m$ dimensional additive 
noise. The principle frame diagram is shown below (Fig. 1) [5].

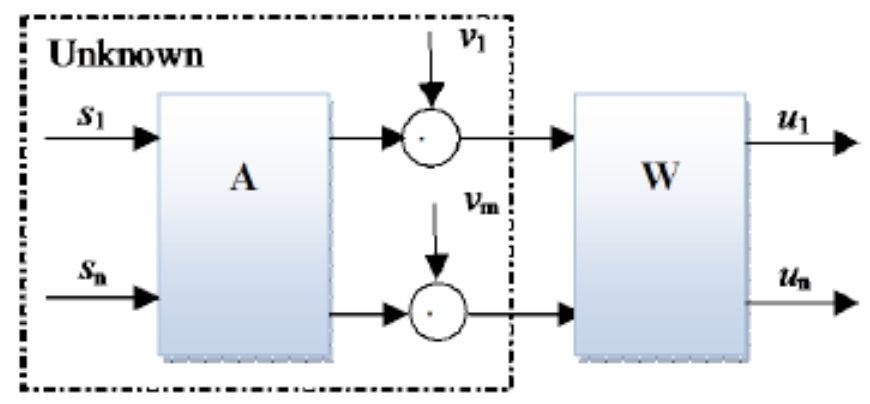

Fig. (1). Blind source separation linear model.

By using a kind of learning algorithm to get the separation matrix $\boldsymbol{W}$, the formula (2) is set up:

$u(t)=W x(t)$

In the formula (2), $u(t)=\left[u_{1}(t), u_{2}(t), \ldots, u_{n}(t)\right]^{T}$ is $n \times 1$ dimensional estimated signal vector, and if global matrix $\boldsymbol{G}=\boldsymbol{W} \boldsymbol{A}=\boldsymbol{I}$ ( $\boldsymbol{I}$ is $n \times n$ dimensional unit matrix $)$, then $u(t)=s(t)$, that is the objective of the source signals is recovered.

\section{VARIABLE STEP SIZE NATURAL GRADIENT ALGORITHM}

The natural blind source separation algorithm was represented by Amari, Cichok [6] based on the mutual information (MI) theory.

$J(k)=-\frac{1}{2} \log \left|\operatorname{det}\left(W^{T} W\right)\right|-\sum_{i=1}^{n} \log p_{i}\left(u_{i}(k)\right)$

In formula (3), $p_{i}\left(u_{i}(\mathrm{k})\right)$ is the probability density function of $u_{i}(k)$ and the separation matrix $\boldsymbol{W}$ is renewed according to the formula (4) [7].

$$
W(k+1)=W(k)+\eta(k+1)\left[I-f(u(k)) u^{T}(k)\right] W(k)
$$

In the formula (4), I is a unit matrix, $k$ expresses discrete time index, $f(\cdot)$ is nonlinear singular function for the estimation signal., $\eta$ is step size $(\eta>0)$, the change quantity of $W(k)$ is the natural gradient based on Riemannian geometry (differential geometry) and comparing Euclidean geometry (plane geometry), the natural gradient uses the Riemann structure parameter space to adjust the direction of the gradient search, which has better convergence $[8,9]$.

And $f(u(k))$ meets the formula (5).

$$
\begin{aligned}
& f(u(k))=\left[f_{1}\left(u_{1}(k)\right), f_{2}\left(u_{2}(k)\right), \ldots, f_{n}\left(u_{n}(k)\right)\right]^{T} \\
& f_{i}\left(u_{i}(k)\right)=-\frac{\partial \log p_{i}\left(u_{i}(k)\right)}{\partial u_{i}(k)}
\end{aligned}
$$

In order to facilitate the discussion, this article adopted crosstalk error to measure the blind source separation effect. The aim of signal blind source separation algorithm is to get a generalized permutation matrix from the mixed-separation composite matrix as far as possible. Therefore, in order to evaluate the performance of a kind of blind source separation algorithm, the reasonable method is adopted to find the difference between the actual mixed-separation matrix and generalized permutation matrix. The definition of blind source separation algorithm performance index is listed in the formula below $(6)[10,11]$.

$$
E=\frac{1}{n(n-1)} \sum_{i=1}^{n}\left\{\left(\sum_{k=1}^{n} \frac{\left|g_{i k}\right|}{\max _{j}\left|g_{i j}\right|}-1\right)+\left(\sum_{k=1}^{n} \frac{\left|g_{k i}\right|}{\max _{j}\left|g_{j i}\right|}-1\right)\right\}
$$

From the formula (6), it can be seen that the performance index is a number no more than zero, that is, $E \geq 0$; however, only when $\mathrm{g}$ is a generalized permutation matrix, $E=0$. But in fact, $E$ is not equal to zero and when the index is more close to zero, the separation algorithm performance is better.

The adaptive natural gradient algorithm has two important features: (1) the algorithm is not affected by the influence of the mixed matrix; (2) the algorithm prevents separation matrix $\boldsymbol{W}$ into a singular matrix and accelerates the rate of convergence of the algorithm [3]. The purpose of blind source separation algorithm is to search the optimal matrix $W_{\text {opt }}$ in formula (4), which makes the final formula (7) lose its realization.

$W(k+1)=W(k)=W_{o p t}, k \rightarrow \infty$

The aim of adjusting any adaptive system parameter is to achieve the following two best integration points of the two performance indexes: (a) the rate of convergence; (b) the steady-state error. Therefore, the purpose of any timevarying step size processing is to increase the step size into a greater stable value, in order to achieve the fastest convergence. And when the best convergence point reaches the neighborhood area, accordingly, the step size and the offset error can be reduced.

When using natural gradient method to realize the blind source separation, it is very important to choose a step size. In order to solve the above contradictions, the study used a kind of variable step adaptive blind source separation algorithm considering the rate of convergence and steady-state performance and compared it with the traditional natural gradient algorithm. In the step size selection, formula (8) was used to update the step size [12].

$\eta(k+1)=\eta(k)-\rho \frac{\partial J(k+1))}{\partial \eta(k)}$

In formula (8), $\rho>0$ is a small number. The matrix inner product is introduced and $\langle D, E\rangle=\operatorname{trace}\left(D E^{T}\right)$ $=\operatorname{trace}\left(D^{T} E\right)$ is defined, $\left.<\cdot, \cdot\right\rangle$ expresses the inner of matrix, trace $(\cdot)$ expresses the trace of matrix. $D$ and $E$ is a $m \times n$ dimensional matrix and because of the complexity of matrix calculus, the definition of inner of matrix is applied. The adaptive variable step size iteration algorithm can be obtained from formula (9).

$$
\begin{aligned}
& \eta(k+1)=\eta(k)-\rho \operatorname{trace}\left\{f(u(k+1)) x^{T}(k+1) W^{T}(k)\right. \\
& \left.\times\left[I-u(k) f^{T}(u(k))\right]\right\}
\end{aligned}
$$




\section{SIMULATION OF SIGNAL BLIND SEPARATION}

The algorithm is based on the natural gradient and on the step size adaptive variable step improvement, therefore theoretically, the convergence speed at the start of the algorithm convergence is faster than an ordinary natural gradient algorithm; however, as time goes on, the length factor decreases gradually, while making the steady-state error smaller and promoting the performance. As a result, the improvement algorithm simulation is carried out.

In order to verify the algorithm validity in noise signal blind source separation, two actual signals are selected, the sampling frequency is $5000 \mathrm{~Hz}$ and the data size is 1000 . In order to verify the effectiveness of the algorithm in the case of noise, we added a group of white noise signal; the source signals are listed below in Fig. (2a-b) and the noise signal waveform in Fig. (2c). The source signal power spectrum is shown in Fig. (3).
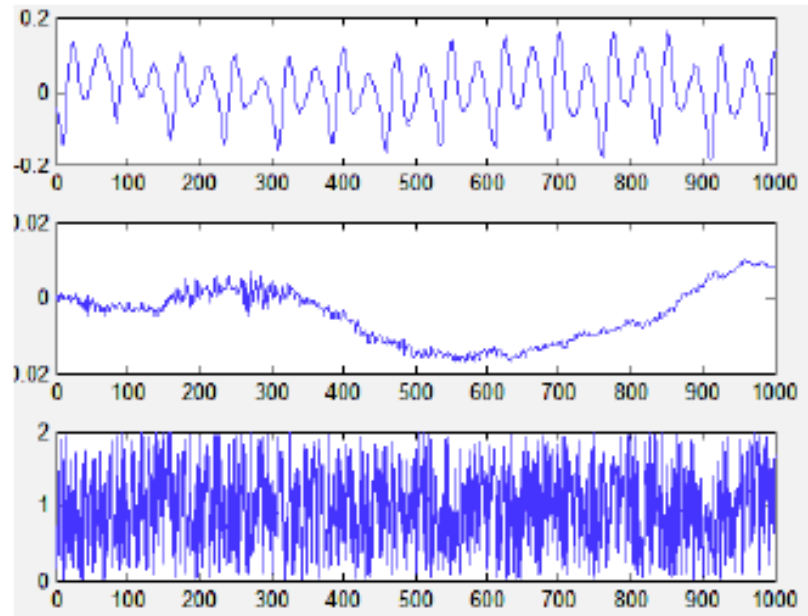

Fig. (2). Waveform of source signal.
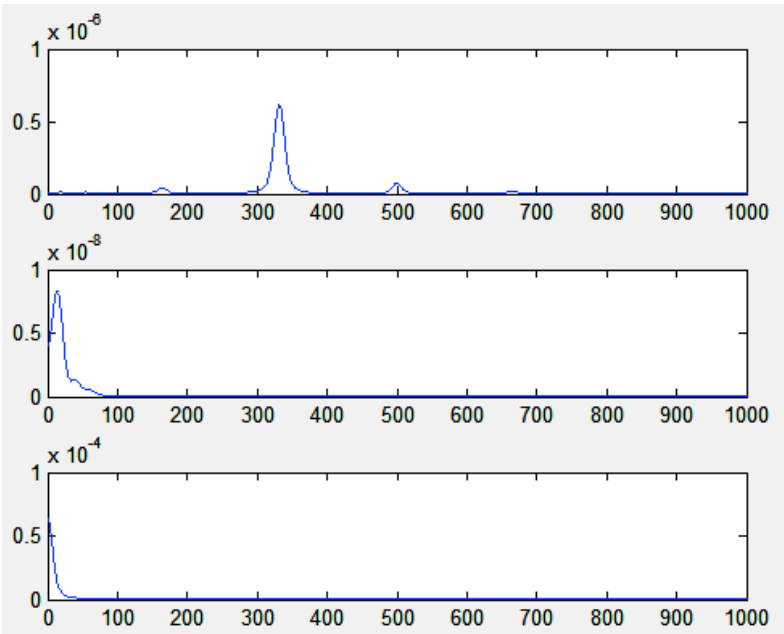

Fig. (3). Spectrum of initial signal.

To make the signal blind mixed, random mixing matrix $\boldsymbol{A}$ is selected.

$$
A=\left(\begin{array}{ccc}
0.5735 & -0.2839 & 0.2012 \\
0.0858 & -0.5223 & -0.9205 \\
-0.2089 & 0.7215 & 0.4789
\end{array}\right)
$$

After mixing the source signal and the noise signal, the observation signal waveform is desultorily shown below in Fig. (4). The signals cannot be traced from which a mixed signal evolves. From the spectrum of observation signal, it can be seen that due to each group of signalsblind mixed, each group of observation signals is mixed with other signals' frequency characteristic, as shown in Fig. (5). Therefore, through observing the blind mixing signals' spectrum, the real signals' detailed information cannot be obtained.
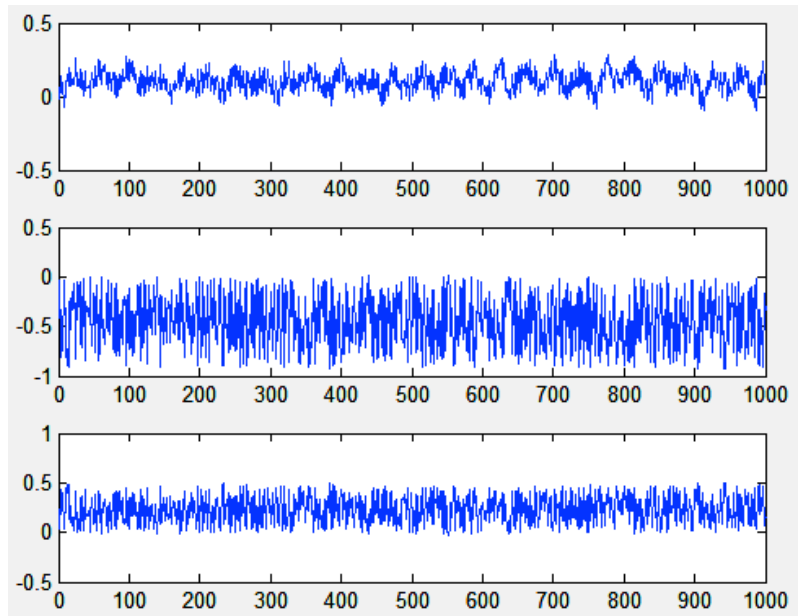

Fig. (4). Observation signal of blind mixed.
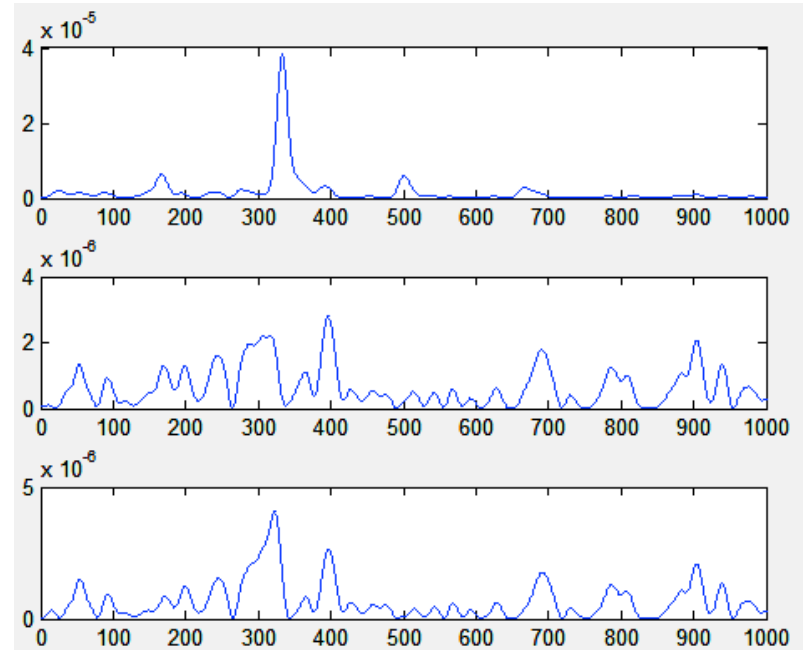

Fig. (5). Frequency spectrum of blind mixed signals.

In view of equal variability of the natural gradient algorithm, the performance has nothing to do with mixture style of the source signals, and it takes a kind of on-line algorithm to adjust the value of separation matrix according to the change of mixed matrix. This study completed blind source separation based on the rule of minimum mutual information natural gradient algorithm. Since the traditional natural gradient algorithm uses the fixed step size, at the beginning of the iteration of the algorithm, if the step size selection is too small, it makes the rate of convergence of the algorithm too slow, but if the step size selection is too large, it makes the algorithm's rate of convergence very fast, so we used a kind of adaptive time-varying step size to have a better effect. In this study, we used the variable step size of the iterative 

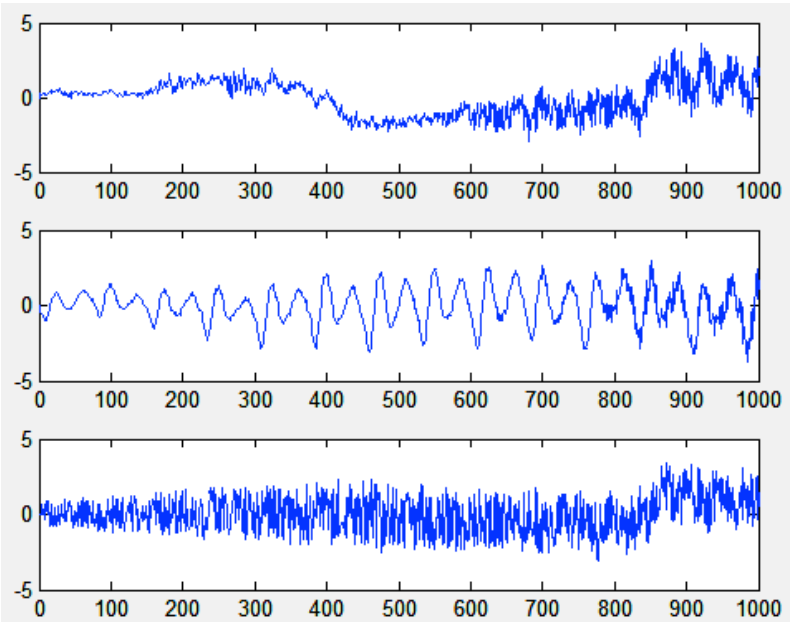

(a) $\mu_{0}=0.005, \rho=10^{-7}$
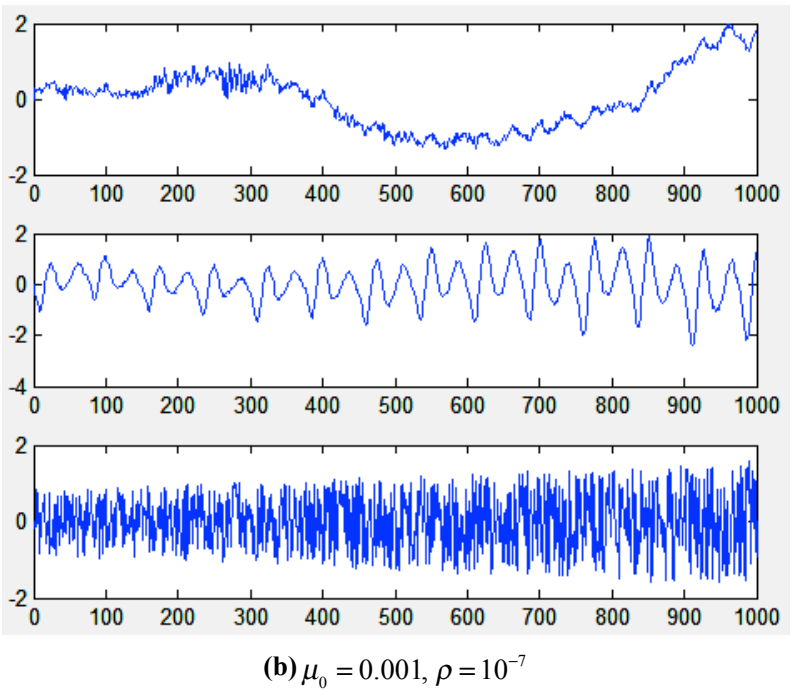

Fig. (6). Separation signal waveform using variable step size natural gradient BSS algorithm.

formula (9), completed the separation and identification of the mixed signal. In formula (9), the nonlinear function of $f(y)$ takes tanh $(y)$. Before separation, we carried out data meaning and bleaching processing [13]. The initial step size $\mu_{0}=0.005, \mu_{0}=0.001, \rho=10^{-7}$ and separation signal were selected respectively as shown in Figs. (6a) and (6b).

In Fig. (6b), the source signals are better restored, which shows that even using the same algorithm, the step size selection is also very important. By taking spectrum in Fig. (6b) and comparing it with the source signal spectrum in Fig. (3), it can be seen that Fig. (6b) separates the blind mixed signal accurately. As the blind source separation has uncertain characteristics, the separation signals have different order as compared with the source. The separation signal better maintains the characteristics of the source signals, with the error being little. As the blind mixed signal contains white noise and other types of noise signals, therefore, in Fig. (7), the separation signal is shown to contain other frequency components, but the value is very small as compared with the source signal.

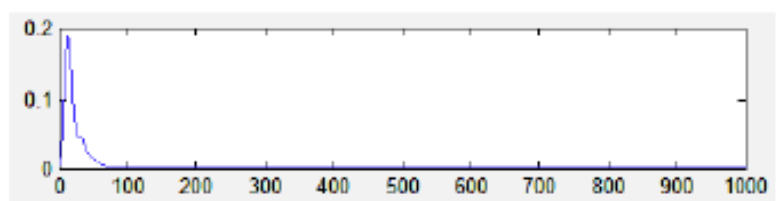

(a)
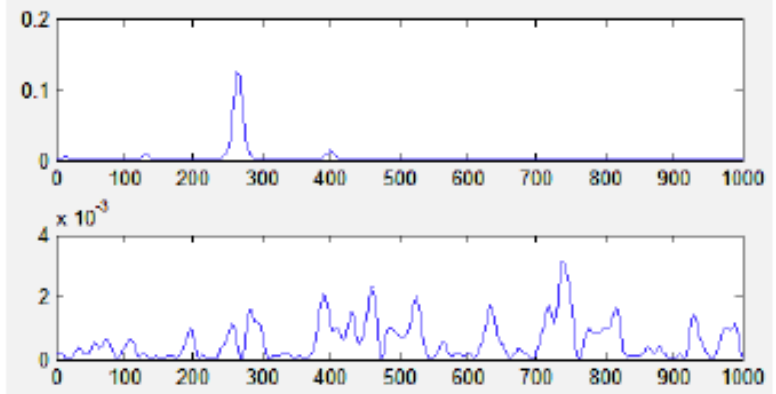

Fig. (7). Frequency spectrum of separation signal.

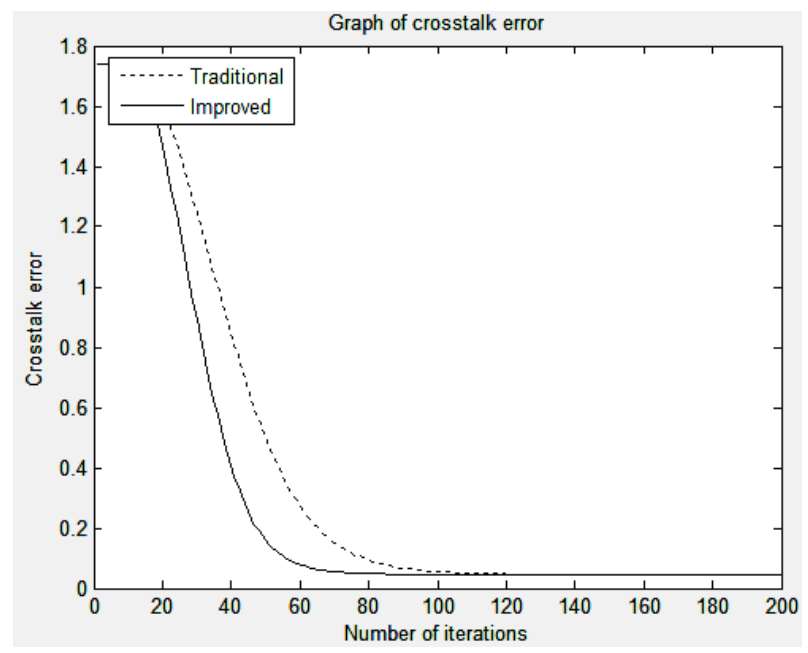

Fig. (8). The graph of crosstalk error index.

From Fig. (6), it can be seen that when choosing a large step size, the algorithm has faster rate of convergence, but steady state performance is poor and the signal error is larger; however, when choosing a small step size, the steady state performance is good and the algorithm can separate source signals more accurately, but the convergence is slower. Therefore, in case of carrying the signal blind source separation, the choice of reasonable step size greatly improves the recognition quality and the method of selecting the step size is the key to improve the effect of separation.

In algorithm performance evaluation, the algorithm performance can be compared with the traditional algorithm and the improved algorithm in virtue of the crosstalk error index. Fig. (8) shows a comparison diagram between the traditional algorithm and improved algorithm in iteration times of 200. The crosstalk error calculation formula is shown as formula (6) in section 3.

The algorithm performance can be concluded with respect to the graph of crosstalk error. For example, the algorithm can achieve faster convergence, and with the small steady-state error, the algorithm's performance is better. In order to blindly pursue the algorithm's convergence speed, bigger steady-state error can be brought, which is not an op- 
tion, and the two indicators between steady-state error and convergence speed should be balanced so that the algorithm realizes its application in signal processing.

\section{CONCLUSION}

This study used the gradient type blind source separation algorithm to complete the noise signal blind source separation. Comparing with Fast ICA batch algorithm, the gradient type algorithm has equal variability, which is a kind of online algorithm and can adjust the value of separation matrix according to the change of mixed matrix. We adopted the adaptive variable step size natural gradient algorithm for better conducting the separation of signals. The adaptive variable step size natural gradient algorithm can balance the contradiction between the convergence speed and the steadystate error. As a result, it is more suitable for the complicated blind separation. However, as the gradient algorithm has much larger computation than the batch processing algorithm, the separation speed is slower than the Fast ICA algorithm. When signal blind source separation is to be conducted, according to a specific situation, the characteristics of different blind separation algorithms should therefore be combined to implement an effective separation.

\section{CONFLICT OF INTEREST}

The author confirms that this article content has no conflict of interest.

\section{ACKNOWLEDGEMENTS}

This paper is supported by Basic Research Project in Shanxi Province (Natural), No. 2013011019-6. And Science and Technology Development Plan Project (Industrial) in Shanxi Province, No. 20140321003-02.

\section{REFERENCES}

[1] J. Ma, Y. Niu, and H. Chen, "Blind Signal Process". Beijing: National Defence Industry Press, 2006.

[2] J. Ma, B. Feng, C. Cheng, S. Zhao, and G. Li, "Application of natural gradient algorithm for blind source seperation to vibration signal processing”, Fire Control and Command Control, vol. 36, no. 4, 154-157, 2011.

[3] X. Wang, and Z. Zhi "Speech blind separation algorithm based on nautral gradient", Electroacoustic Technology, vol. 33, no. 1, pp. 63-64, 2009.

[4] X. Zhang, and Z. Bao, "Blind signal separation", Acta Electronica SinicA, vol. 29, pp. 1766-1771, 2001.

[5] Y. Gao, Y. Li, and B. Yang, "Overview on variable step size techniques for on-line blind source separation", Computer Engineering and Applications, vol. 43, no. 19, p. 75, 2007.

[6] I. S. Amar, and A. Cichocki, "Adaptive blind signal processing neural network approaches", In: Proceedings of the IEEE, vol. 86, no. 10 , pp. 2026-2048, 1998.

[7] S. Sun, J. Zheng, and D. Wu "Research on blind source separation based on natural gradient algorithm", Journal of Air Force Engineering University (Natural Science Edition), vol. 4, no. 3, pp. 5052,2003

[8] S. Amari, "Natural gradient works efficiently in learning", Neural Computation, vol. 10, no. 2, pp. 251-276, 1998.

[9] S. Amari, and S. C. Douglas, "Why Natural Gradient", In: IEEE International Conference on Acoustics, Speech, and Signal Processing, vol. 2, pp. 1213-1216, 1998.

[10] G. Li, and J. Zhang, "Adaptive step-size EASI algorithm based on separating matrix", Ship Science and Technology, vol. 5, no. 28, pp. 69-72, 2006.

[11] S. Xia, T. Zhang, X. Li, and Q. Gao, "Adaptive blind separation algorithm of mixed image based on variable step-size", Journal of Data Acquisition and Processing, vol. 26, no. 2, pp. 156-161, 2011.

[12] Z. Li, and Z. Liyi, "A new adaptive step size algorithm for blind source separation", Modern Electronic Technology, vol. 28, no. 24, pp. 96-97, 2005.

[13] X. Liu, L. Ma, and X. Tan, "Integrated blind source separation algorithm with natural gradient based on whitening process", Journal of Harbin Institute of Technology, vol. 42, no. 7, pp. 10461048, 2010.

\begin{tabular}{lcc}
\hline Received: September 22, 2014 & Revised: November 30, 2014 & Accepted: December 02, 2014 \\
(C) Liu Fangyu; Licensee Bentham Open. &
\end{tabular}

This is an open access article licensed under the terms of the Creative Commons Attribution Non-Commercial License (http://creativecommons.org/licenses/by-nc/4.0/) which permits unrestricted, non-commercial use, distribution and reproduction in any medium, provided the work is properly cited. 